\title{
ESTUDIO DEL NIVEL OPERATORIO DE ESTUDIANTES UNIVERSITARIOS
}

CELIA DIBAR URE, M. y R.P. QUEIROZ, G. Universidad Federal Fluminense, Brasil

\section{SUMMARY}

The shadows task proposed by J. Piaget was chosen, after preliminary interviews, to study the level of congnitive operations of university freshmen at Universidad Federal Fluminense, Brazil. We interviewed 82 students of engineering, chemistry and physics using clinical, individual interviews. We disctuss the results and possible applica* tions to the teaching of physics at this level.

\section{INTRODUCCION}

Preocupados con el alto indice de reprobación en las físicas elementales encontrado en nuestra universidad (fenómeno que es por otra parte un problema de todas las universidades brasileras) y también con la dificultad que los alumnos tienen para resolver problemas que no sean mera substitución en fórmulas, comenzamos un estudio de la forma de razonar de estos alumnos. Seguimos para ello, en líneas generales, la teoria desarrollada por la escuela de Ginebra de epistemología genética.

Los primeros trabajos sobre adolescentes (Inhelder y Piaget, 1955) muestran que los jovenes de Ginebra llegan a las operaciones formales o al razonamiento llamado hipotético-deductivo en la adolescencia, pero el propio Piaget posteriormente cuestiona la generalidad de esta observación (Piaget 1972).

Trabajos más recientes (McKinnon 1971, Griffiths 1976, Schircks y Laroche 1970) con jóvenes universitarios y adultos en cursos de especialización muestran que un porcentaje relativamente grande de estudiantes adultos no llegaron al estadio de las operaciones formales. Estas son indispensables para la comprensión de disciplinas científicas a nivel universitario pues un alumno no formal, por ejemplo, no trabaja satisfactoriamente con proporciones, no sabe verificar hipotesis, ni hacer sistemáticamente una combinatoria. Tales conclusiones tendrian entonces importantes consecuencias pedagógicas independientemente de tratarse del estudio de estructuras fundamentales de la epistemologfa genética.
En forma paralela a este estudio y usando sus resulta. dos, intentamos desarrollar una forma más rápida de evaluar el nivel de operaciones usando para ello una version escrita de ciertas tareas piagetianas que permiten esta adaptación (Dibar Ure, Queiroz 1979).

\section{ENTREVISTAS PRELIMINARES, LAS PRUEBAS Y LAS RESPUESTAS DE LOS ALUMNOS}

Esta investigación tuvo dos fases preliminares. En una primera fase, dedicada a caracterizar el problema, se entrevistaron a unos 30 alumnos usando pruebas de péndulo, flotación de cuerpos, cuantificación de las probabilidades y correlaciones (Inhelder y Piaget 1955, Piaget 1951). Este trabajo ha sido publicado separadamente (Dibar Ure 1982).

Una segunda etapa consistió en entrevistar a 30 alumnos con pruebas de balanza de brazos, flotación de cuerpos, combinaciones químicas, cuantificacion de las probabilidades, flexibilidad de varillas y sombras (Inhelder y Piaget 1955, Piaget 1951). Se estudiaron en esta fase las pruebas propuestas y las respuestas de los estudiantes y decidimos usar en este trabajo final, la tarea de sombras en la forma de entrevistas clínicas individuales. Este método, muy flexible y que permite acompanar en detalle los razonamientos de los suje tos, ha sido usado extensamente por la escuela de Ginebra (Inhelder 1974).

Esta fase preliminar fue muy importante para ajustar 
las tareas propuestas y las entrevistas a nuestra población. Gruber y Vonèche (1976) alertan sobre las dificultades que se pueden encontrar en el estudio de adultos que pueden llegar casi a una "parálisis operatoria» al ser observados. Encontramos que no fue este el caso con los aduitos de nuestra muestra, que se sintieron en general cómodos con las tareas propuestas.

En lo que se refiere a las respuestas concluimos que: 1) Los razonamientos (en especial nos interesaban los errados) de estos jóvenes adultos eran asombrosamente parecidos a los de los adolescentes ginebrinos lo cual sugiere la conveniencia de aplicar la epistemologia genética para explicar cómo piensan los nuevos universitarios. 2) Algunas tareas provocaban tantas respuestas memorizadas, de tipo escolar, con tanto vocabulario "pseudo técnico" que fue imposible en muchos casos evaluar qué operaciones mentales estaban involucradas. Este era el caso de Péndulo, y en menor escala de Baianza, que fueron abandonadas como pruebas luego de algunas tentativas.

El análisis de las tareas usadas por Inhelder y Piaget en su estudio del pasaje de la lógica del niño a la lógica del adolescente debe comenzarse recordando que, como en la mayor parte de la obra de Piaget, el interés es fundamentaimente epistemológico. Se cuestiona allí la relación entre el éxito de los jovenes adolescentes en la resolución de ciertas tareas y la aparición de dos estructuras mentales relacionadas entre ellas: la red (de la lógica proposicional en particular) o conjunto de todos los posibles subconjuntos que también está asociado al uso de combinaciones y permutaciones en otros contextos, y el grupo que contiene las dos reversibilidades, el grupo INRC (Piaget 1971), (Inhelder y Piaget 1955).

Por lo tanto los autores de «De la lógica del niño a la lógica del adolescente» (Inhelder y Piaget 1955) no están interesados con el hecho que algunas tareas involucren más que el esquema formal que pretenden ilustrar (por ejemplo flotación es una prueba muy interesante pero allí se encuentran muchos factores que influyen y aparecen entonces diferentes estrategias de los alumnos para lidar con material complejo) (M.C. Dibar Ure 1982).

En la obra citada también se dejan pasar varios errores de física, de los cuales daremos 2 ejemplos:

I) Al discutir la prensa hidráulica, los autores hablan, sin mucha precisión de la "reacción" del líquido y también de la presión como teniendo diversas orientaciones.

2) En el capftulo XIV intitulado «Fuerza centrifuga y compensaciones» en ningún momento se menciona cual es el referencial usado lo que puede incidir en el error habitual entre los alumnos (y algunos profesores!) de considerar las fuerzas centrffugas como reales. Se estudia el movimiento de varias esferas de distinto tamafo colocadas sobre un disco giratorio. Se pide al niño que prevea qué esfera comenzará a moverse antes, cuando es de prever que estas esferas comenzarán todas al mismo tiempo a rodar sin deslizar.

En el análisis ffsico del problema, partiendo de la expresión $\mathrm{f}=\mathrm{m} \mathrm{w}^{2} \mathrm{r}$ para la fuerza centrífuga (donde $\mathrm{w}$ es la velocidad angular del disco giratorio, $m$ la masa de la esfera y $r$ la distancia de ésta al centro del disco) se saca como conclusión que como w es constante saldrán antes las esferas de mayor $m$ y a mayor distancia. Pero esto es asociar la fuerza al instante de partida en vez de a la aceleración, un error del tipo de los "preconceptos aristotélicos» estudiados por varios autores (Clement 1982, Viennot 1979) que asocian fuerzas a velocidades en vez de aceleraciones.

Además de las experiencias de péndulo, cuantificación de las probabilidades, correlaciones y flotación discutidas en un artículo anterior (M.C. Dibar Ure 1982), concluimos que las pruebas de combinaciones químicas, sombras y flexibilidad resultaron muy adecuadas a la población universitaria.

Por sus características, dejamos combinaciones químicas, flexibilidad y probabilidades para ser incluidas en una versión escrita (Dibar Ure y Queiroz 1979) y elegimos la prueba de sombras para las entrevistas finales. Además de resultar inter esante y nueva para los alumnos entrevistados en esta fase preliminar, no presentaba errores de física en su formulación ni provocaba respuestas escolarizadas.

También permitía simultáneamente evaluar el uso de hipótesis proporcionales (relacionadas con la aparición del Grupo INRC) y estudiar la forma de verificar hipótesis (asociado a la lógica proposicional).

\section{ENTREVISTAS FINALES. DESCRIPCION DE NUESTRA TAREA Y DE LA MUESTRA}

A continuación describiremos breveménte la entrevista de sombras y tambien presentaremos algunas respuestas típicas de alumnos para ejemplificar la clasificación realizada.

El equipo utilizado es muy simple consistiendo de una fuente luminosa montada en un extremo de una vara con agujeros equiespaciados y una pantalla en el otro extremo (ver figura 1). Esta permite montar, en posiciones variables, cuatro discos de diámetros de 5,10 , 15 y $20 \mathrm{~cm}$ respectivamente.

Se le pide al alumno que obtenga sombras iguales en la pantalla usando los discos de diferentes tamafios.

Luego de familiarizarse libremente con el material, se le da un disco en una determinada posicion (por ejemplo el disco de $10 \mathrm{~cm}$ en la $8^{\circ}$ posición a partir de la fuente de luz) y se le pide que ubique uno o más de los restantes para obtener la misma sombra.

Luego de algunas tentativas, comenzábamos a pedir que predijese la posición antes de experimentar, y también pediamos una justificativa. 


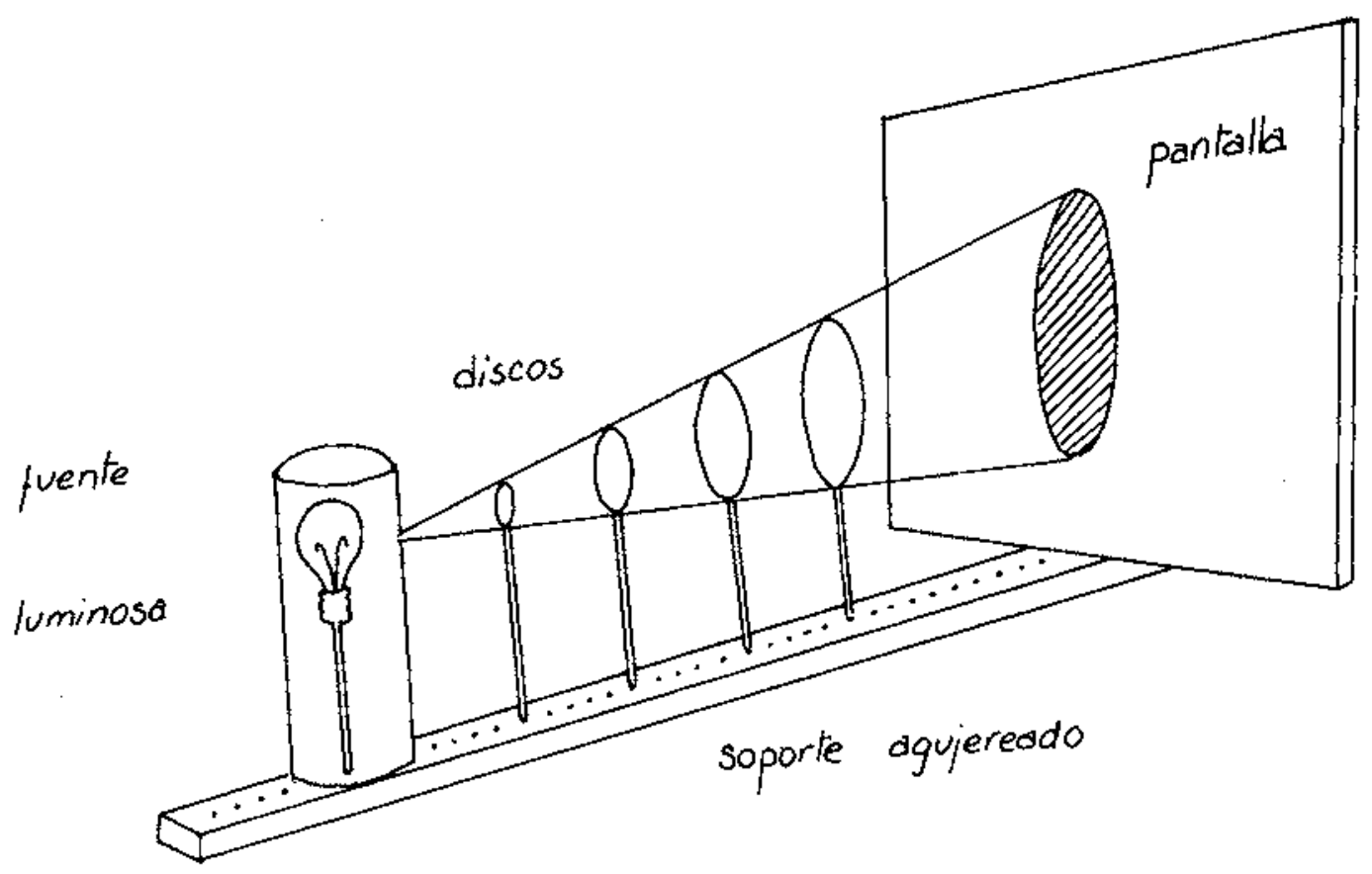

FIG. 1 - Esquema experimental de la prueba de sombras

Como es habitual en las entrevistas clínicas, se variaban los ejemplos hasta que quedase claro para el experimentador como razonaba el alumno. Al final de la entrevista se pedía al alumno un resumen de lo que había encontrado, con la indicación que fuese suficientemente claro como para que un comparfero que no ha. bia hecho la experiencia pudiese prever bien las posiciones inmediatamente.

La entrevista duraba unos 45 minutos y las notas to. madas durante ésta eran en seguida estudiadas para permitir la evaluación del nivel operatorio. 32 de estas fueron tomadas y juzgadas por 2 jueces independientes usando los niveles descritos por Inhelder y Piaget con algunas modificaciones que discutimos a continuación con los ejemplos:

Nivel II B $_{\mathbf{B}}$ : Inhelder y Piaget clasifican dentro del subnivel $\mathrm{II}_{\mathrm{B}}$ (operaciones concretas bien establecidas, $\mathrm{O}$ equilibradas) dos comportamientos diferentes que también encontramos en nuestros jóvenes adultos: a) co. rrespondencias cualitativas claras entre el tamafto de las sombras y el tamaño y la posición de los discos y noción de que distancia a la fuente y tamaño del disco se compensan. Aún frente a la insistencia del entrevistador para dar previsiones más precisas, estos alum. nos se rehusan a dar previsiones numéricas. Ejemplo:

\section{A.M., 20 años, (alumno de) Ingeniería}

"Cuanto más cerca (de la fuente) mayor es la sombra» (mantiene este tipo de respuestas aún frente a insisten. cia de precisar más su previsión). b) $50 \%$ de los alumnos clasificados como concretos en nuestra muestra luego de hacer compensaciones cualitativas, intentaron predecir con cálculos numéricos pero usando diferencias en vez de proporciones. Por ejemplo:

\section{Ca, 19 años, Ingeniería Química}

Trata de mantener la misma distancia entre los discos que habia encontrado en su primer ejemplo $(5 \mathrm{~cm}$ en $\mathrm{l}, 10 \mathrm{~cm}$ en $9,15 \mathrm{~cm}$ en 14 y $20 \mathrm{~cm}$ en 18). Así cuando se le pidió para prever donde colocar el disco de $20 \mathrm{~cm}$ de diámetro cuando el de $10 \mathrm{~cm}$ estaba colocado en la $8^{a}$ posición, predijo que debería colocarse en la posición 17 (manteniendo asi la diferencia anterior de 9 posiciones). Aún cuando no consigue resultados satisfac. torios insiste con este tipo de propuestas hasta el fin de la entrevista.

Entre los primeros 24 alumnos entrevistados encontra* mos 4 casos en que los jueces quedaron inciertos si clasificar las respuestas como perteneciendo al nivel $\mathrm{II}_{B}$

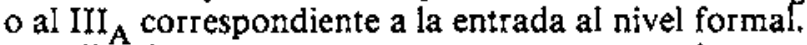
Estudiando estos casos, encontramos que tenían una característica común que nos pareció asociada a la transición entre dichos niveles: todos estos alumnos usaban proporciones sólo cuando las fracciones eran mitades. En los otros casos usaban diferencias constantes o predicciones cualitativas. Decidimos clasificarlos como casos intermedios entre los niveles $\mathrm{H}_{\mathrm{B}}$ y $\mathrm{III}_{\mathrm{A}}$. 


\section{Ce. $\mathrm{Xa}, 23$ años, Física}

Cuando se le da el disco de $10 \mathrm{~cm}$ en la $8 \mathrm{a}$. posición, preve que el de $20 \mathrm{~cm}$ caerá en 25 o 26 (cualitativo) y preve que el đe $15 \mathrm{~cm}$ caerá en la posición 17. Aproximadamente en el medio de los dos porque el diámetro también está en el medio. Y más tarde cuando se le da el disco de $5 \mathrm{~cm}$ en la posición 7, no sabe bien donde colocar el de $15 \mathrm{~cm}$ "si fuese el de 10 sería el doble de la distancia, como es el 15 será mayor, $2 \circ 3$ posiciones más». Para el de 10 preve entonces «será a la mitad de la distancia (entre el de 15 y el de 5)».

Este tipo de razonamientos con mitades y dobles antes de poder realizar correctamente otras proporciones también se ve en las entrevistas de cuantificación de probabilidades del propio Piaget (Piaget 1951).

Nivel III ${ }_{A}$ : corresponde, como ya hemos indicado, a la entrada al nivel formal. Los sujetos tratan de usar proporciones entre las distancias y los diámetros de los discos en algunos casos, pero sin generalización a todos los casos posibles. Piaget indica que sólo aquí los estudiantes comienzan a medir las distancias a partir de la fuente luminosa, mientras que los alumnos del nivel $\mathrm{II}_{B}$ las median a partir de la pantalla pues prefieren las correspondencias directas. No encontramos estos resultados con nuestros alumnos: muy pocos del nivel $\mathrm{II}_{\mathrm{B}}$ median las distancias desde la pantalla, ya que la mayoria, como comentamos, estaban preocupados con la distancia entre los discos que trataban de mantener constante. En cambio, varios alumnos que intentaban proporciones y que clasificamos como pertenecientes al nivel $\mathrm{III}_{\mathrm{A}}$, intentaron proporciones directas midiento desde la pantalla.

Encontramos que los sujetos del nivel $\mathrm{III}_{\mathrm{A}}$ generalmente intentaban proporciones erradas y a veces las descartaban, pero luego las intentaban de nuevo, como si no fuese claro para ellos que un contraejemplo basta para descartar una hipótesis, o, como escribe Inhelder y Piaget (1955). "No busca aún la ley general como un sistema de relaciones necesarias y suficientes para explicar el resultado obtenido'.

Veamos un ejemplo de alumnos de este nivel:

\section{V.F., 22 años, Química}

Trabaja correctamente con mitades, por ejemplo coloca el disco 15 en la mitad entre el de 10 y el de 20.

Dado el disco de $10 \mathrm{~cm}$ en la $8 \mathrm{a}$. posición, predice que el de 20 caerá en la posición 15 explicando «la distancia del disco de $10 \mathrm{~cm}$ será $2 / 3$ de la distancia del de $20 \mathrm{~m}$. Para colocar el disco de $10 \mathrm{~cm}$ dado el de $5 \mathrm{~cm}$ en la posición 12, explica "tenemos que dividir la distancia (a la pared) por 2 , si fuese el de $15 \mathrm{~cm}$ la dividiría por 3 \%.

No parece preocuparse cuando sus predicciones no corresponden con la situación correcta.
El nivel III ${ }_{B}$ corresponde al razonamiento formal bien establecido o uequilibrado». Según Inhelder y Piaget (1955) «la construcción se halla de entrada subordinada a una hipótesis a la vez explicativa y general... Este esquema explicativo implica la proporcionalidad y se aplica (...) a cualquier distancia».

Encontramos que nuestros sujetos verificaban exhaustivamente las hipótesis. A veces comenzaban desde hipótesis cualitativas o de diferencias, pero llegando a proporciones. En la mayoría de los casos llegaban a la ley correcta, pero también clasificamos como $\mathrm{II}_{\mathrm{B}}$ a un alumno que no consiguió llegar a una ley correcta pues comenzó midiendo las distancias a partir de la pantalla, pero trabajó concienzudamente en destacar todas sus hipótesis proporcionales incorrectas.

Ejemplos de $\mathrm{III}_{\mathrm{B}}$ :

\section{D.S., 18 años, Ingeniería}

Trabaja con mucho cuidado desde el comienzo. Cuando se le da el disco de $10 \mathrm{~cm}$ en la posición $8 \mathrm{a}$. y se le pide de colocar el disco de $20 \mathrm{~cm}$, dice ucomparando el tamaño del objeto, tengo que encontrar una distancia donde el ángulo (se refiere a cono de la luz) tenga una abertura del objeto de $20 \mathrm{~cm}$ (sic). Predice la posición 16 (correcta) y exclama al verificarlo «perfecta», Después de una situación experimental más, encuentra la ley general correcta.

\section{M.V., 19 años, Ingeniería}

En el primer ejemplo intenta una hipótesis aditiva ( 7 de distancia entre los discos). Luego predice distancia doble para radio doble $(10 \mathrm{~cm}$ en $8 \mathrm{a}$. posición, $20 \mathrm{~cm}$ a $16 \mathrm{~cm}$ ) y luego llega a la ley general, explicando «la proporción de los radios es la misma que la de las distancias" y la usa prediciendo correctamente una nueva situación, usando lápiz y papel para hacer los cálculos.

\section{RESULTADOS Y DISCUSION}

Elegimos nuestra muestra de estudiantes que cursaban la primera física elemental de forma que tuviese aproximadamente el mismo porcentaje de ingenieros, quimicos y físicos, para quienes se ofrece este curso.

33 de los 82 estudiantes entrevistados (excluyendo los 4 primeros casos de intermediarios que ya discutimos) fueron clasificados independientemente por 2 jueces. El coeficiente de correlación entre las clasificaciones de los 2 jueces (Siegel 1975 ) resultó $r=0.994$, tomando los estadios de $\mathrm{II}_{\mathrm{B}}$ a III $\mathrm{I}_{\mathrm{B}}$ como ordenados de 1 a 4 y usando

$$
r=\frac{\sum x y}{\sqrt{\sum\left(x^{2}\right) \sum\left(y^{2}\right)}}
$$


donde ' $\mathrm{x}$ ' representa la posición dada a un estudiante por el juez I y ' $y$ ' por el juez 2 y la suma se extiende sobre los 33 estudiantes. Podemos confiar entonces $(n=31, p \quad 0.999)$ que las evaluaciones de los jueces están altamente correlacionadas.

La entrevista con el resto de los estudiantes fue entonces evaluada por cualquiera de los 2 jueces.

En la Tabla I mostramos los porcentajes de estudiantes en los diferentes niveles operatorios, por carrera. También incluímos a modo de comparación, datos de Mckinnon y Renner (1971), Griffiths (1976) y Schircks y Laroche (1970) que usaron entrevistas similares con adultos jóvenes. Mckinnon y Renner entrevistaron alumnos de ler. año de la universidad con tareas de conservación de volumen, reflexión mecánica en una pared, flotación de cuerpos, péndulo y probabilidades. Clasificaron los comportamientos según 3 niveles operatorios: concreto, intermedio y formal. Griffths, que también trabajó con universitarios del ler. año usó la prueba de plano inclinado y sólo clasificó los alumnos en formal y no formal pues enfocó en su estudio el uso de lenguaje (técnico o no técnico).

Schircks y Laroche (1970) entrevistaron adultos que en su mayoria habian abandonado la escuela secundaria cerca de los 14 años y que se encontraban haciendo un curso de especialización para técnicos. La media de edades era 23 años y fueron entrevistados con pruebas de cuantificacion de probabilidades, permutaciones y combinaciones, péndulo, conservación de volumen y curvas mecánicas.

De la Tabla podemos observar que nuestros resultados son de forma general similares a los de los otros autores.
La comparación con McKinnon y Renner no se puede hacer con exactitud porque usan una clasificación ligeramente diferente de la de Piaget, pero podemos no. tar que si tomamos su nivel intermedio como nuestro intermedio más nuestro nivel III $_{\mathrm{A}}$, nuestros mejores estudiantes, los de ingeniería, dan porcentajes muy pa. recidos a los de los alumnos norteamericanos, en cuanto que nuestros estudiantes de física se parecen más a los técnicos franceses.

Los resultados tan diferentes obtenidos para los alumnos de física y quimica comparados con los de inge. nieria no son sorprendentes. En el sistema educacional brasilero, debido posiblemente a características del mercado de trabajo, la competencia para entrar a ingeniería es mayor que en las otras carreras, y naturalmente tiene alumnos de más nivel.

A diferencia de Mckinnon y Renner o Schircks y Laroche, realizamos las entrevistas usando solamente una prueba, pero como ésta fue elegida después de toda una fase preliminar, encontramos que era una tarea espe cialmente adecuada para evaluar simultáneamente 2 habilidades centrales en el estadio formal: manejo de proporciones y verificación de hipótesis.

El número de alumnos de física y química que se en. cuentran debajo del nivel III $_{\mathrm{A}}$ deberia dar una seria ad. vertencia a los profésores universitarios sobre las razones de las dificultades que pueden ser encontradas al enseñar un curso tradicional de física elemental. Se presume muchas veces que los alumnos son capaces de seguir aulas expositivas con demostraciones abstractas cuando vemos que muchos no son capaces de razonar en forma lógica ni de trabajar aún con funciones lineales.

$I A B \perp A \quad I$

\begin{tabular}{|c|c|c|c|c|c|c|c|}
\hline \multicolumn{2}{|r|}{ Nuestros } & \multicolumn{3}{|l|}{ Resultados } & \multicolumn{3}{|c|}{ Otros autores con otras tareas } \\
\hline $\begin{array}{l}\text { Wive } 1 \text { ope- } \\
\text { ratorio }\end{array}$ & $\begin{array}{l}\text { \& Poblacion } \\
\text { totai } N=8 ?\end{array}$ & $\begin{array}{l}\text { Es Estudiantes } \\
\text { de física } N=2 A\end{array}$ & $\begin{array}{l}\text { \% Es tudiantes } \\
\text { química } \mathrm{N}=\mathbf{2} ?\end{array}$ & $\begin{array}{l}\text { FEstugiantes } \\
\text { ingentería } N=35\end{array}$ & $\begin{array}{l}\text { Mckinnon y } \\
\text { Rennei (QS) }\end{array}$ & $\left\{\begin{array}{l}\text { Griffiths } \\
(9976)\end{array}\right.$ & $\begin{array}{l}\text { Schircks y } \\
\text { Larocre (1970) }\end{array}$ \\
\hline$I_{3}$ & 24 & 38 & 18 & 17 & 18 & & 12 \\
\hline I:UT. & 15 & 13 & 23 & 9 & & & 42 \\
\hline $\operatorname{II} I_{n}$ & 41 & 32 & 50 & 44 & & . & 38 \\
\hline $\mathrm{II} \mathrm{I}_{\mathrm{B}}$ & 20 & 17 & 9 & 31 & 32 & & 8 \\
\hline
\end{tabular}




\section{REFERENCIAS BIBLIOGRAFICAS}

CLEMENT, J. 1982, Students preconceptions in introductory mechanics, Am. J. Phys, 501 pp 66-71.

DIBAR URE, M.C. y QUEIROZ, G.R.P. 1979. Nova tentativa para a realizaçao de um teste coletivo para veriffcar como raciocina um adulto (IV Simpósio de Ensino de Física, Río de Janeiro),

DIBAR URE, M.C. 1982, Estudo Preliminar do Nivel Operatório de Estudantes Universitários, Rev. Bras. de Física, Vol, $12 \mathrm{n}^{\circ} 12$ pp. 363 a 374.

GRIFFITHS, D.H. 1976, Physics Teaching: Does it hinder intellectual development?, Am. J. Phys., Vol. $44 \pi^{\circ} 1$, pp. 81-85.

GRUBER, H. y VONECHE, J, 1976, Reflexions sur les operations formelles de la pensée, Arch. Psych. XLIV, $\pi^{\circ} 171$, pp. 45-55.

INHELDER, B. 1974. Apprentissage et Structures de Connaissance, (Paris: PUF).

INHELDER, B. y PIAGET, J. 1955, De la logique de l'enfant a la logique de l'adolescent, (Paris. PUF) (Traducido al castellano por Pajdós, Buenos Aires, 1972).
MCKINNON, J. W. y RENNER, J. W. 1971, Are colleges concerned with intellectual development? Am. J. Pys., Vol. $39, n^{\circ} 9$ pp. 1047-1053.

PIAGET, J. 1951, La Genèse de l'idée de hasard chez l'enfant. (Paris, PUF).

PIAGET, J. 1971, Essai de Logique Operatoire (Paris: Dunod).

PIAGET, J. 1972, Intellectual development from adolescence to adulthood, Human Development, Vol. 15, $\mathrm{n}^{\circ} 1$, pp. $1 \cdot 12$.

SIEGUEL, S. 1975, Estatística näo paramétrica para as cièncias do Comportamento (S. Paulo: McGraw Hill).

SCHIRCKS, A. y LAROCHE, J.L. 1970, Etude des opérations intellectuelles chez les adultes de la promotion supérieure de travail, Travail Humain, Vol. $33, \mathrm{n}^{\circ} .2 \mathrm{pp}$. 99-112.

VIENNOT, L. 1979, Spontaneous Reasoning in Elementary Dynamics, Eur. J. Sci. Educ., Vol. 1, ri ${ }^{\circ}$. 2, pp. 205-221. 